\title{
The Difference between Activation Energies for the Layer Growth and Interdiffusion in an Intermediate Phase Formed in a Poly-Phase Diffusion Couple*
}

\author{
By Yasuhiro Funamizu** and Katsuya Watanabe**
}

\begin{abstract}
The purpose of this study was to account for the origin of the difference between activation energies for the layer growth and interdiffusion in an intermediate phase which was formed in a poly-phase diffusion couple. It was shown that this difference between the two activation energies seems to originate from the temperature dependence of the homogeneity range of the phase. Accordingly, the activation energy of the layer growth $\left(Q_{K}{ }^{2}\right)$ is equal to a sum of the energy of the interdiffusion $\left(Q_{D}\right)$ and that attributable to the temperature dependence of the homogeneity range of the phase $\left(Q_{g}\right)$.

As a practical example, the energy difference for the $\beta$ phase in an $\mathrm{Al}-\mathrm{Cu}$ system was indicated quantitatively. For the $\beta$ phase, $Q_{\mathrm{K}^{2}}$ is $43.6 \mathrm{kcal} / \mathrm{mol}, Q_{D}$ is $27.8 \mathrm{kcal} / \mathrm{mol}$, and $Q_{g}$ is $18.3 \mathrm{kcal} / \mathrm{mol}$. The sum of $Q_{D}$ and $Q_{g}$ is $46.1 \mathrm{kcal} / \mathrm{mol}$, which coincides with $Q_{\mathbf{K}}{ }^{2}$ within the experimental accuracy.

It is also concluded that the activation energy for a layer growth does not coincide with that for an interdiffusion in the phase where the change in the concentration with temperature at that phase boundary is found.
\end{abstract}

(Received May 30, 1973)

\section{Introduction}

In the studies on poly-phase diffusion, there have been many cases with no clear distinction between the activation energy for the growth of an intermediate phase layer and that for the interdiffusion in the phase. Several recent experimental studies on interdiffu$\operatorname{sion}^{(1) \sim(3)}$ have reported that the two activation energies differ considerably from each other. In general, it seems that a clear distinction can be obtained between the two kinds of activation energies. When the growth of an intermediate phase in a diffusion zone depends on the nucleation of the phase or on any conditions of the phase boundary, it is natural to anticipate that these two activation energies have unequal values. However, the present authors would rather like to deal with the case where the growth of the layer obeys the parabolic law, that is, the process is diffusion-controlling, and further the activation

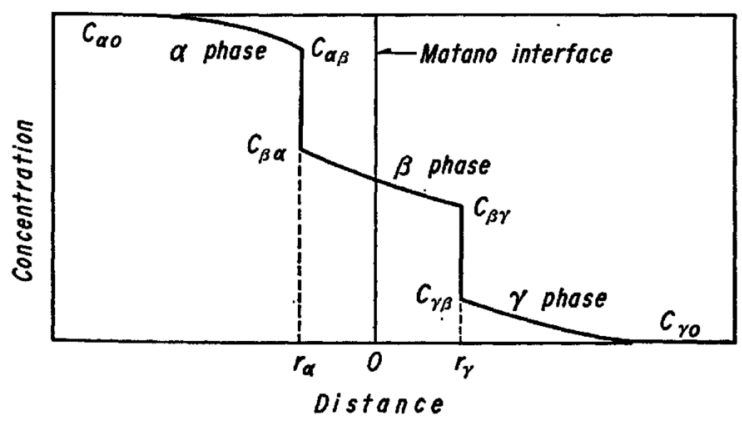

Fig. 1 Schematic concentration-penetration curve for diffusion zone as a result of the interdiffusion between $\alpha$ and $\gamma$ phases.

* This paper was presented at the Autumn Meeting of the Japan Institute of Metals, October, 1972, Nagoya, Japan.

** Faculty of Engineering, Hokkaido University, Sapporo, Japan.

Trans. JIM energy for a layer growth differs from that of an interdiffusion.

It would be significant for a better understanding of the phenomena observed in a poly-phase diffusion to make clear the factors controlling the occurrence of the activation energy difference.

The purpose of this study was to introduce theoretically a quantitative analysis for the energy difference between layer growth and interdiffusion in the phase, and to prove the propriety of the analysis through an experimental work.

\section{Relation between the Activation Energy for Growth of Intermediate Phase Layer and that for Interdiffusion}

Several researchers ${ }^{(4) \sim(6)}$ have studied phenomenologically the growth of an intermediate phase layer formed in a poly-phase diffusion zone and introduced some equations which express the relation between the rate of phase growth and the interdiffusion coefficient. However, it is not necessarily appropriate to discuss quantitatively the relation between the two activation energies by means of these treatments. In this study, based on the above treatments, an attempt was made to make a clear distinction between the temperature dependence of the two factors.

Consider that a $\beta$ phase was formed as a result of the interdiffusion between the $\alpha$ phase and the $\gamma$ phase. The initial compositions of these phases were $C_{\alpha 0}$ and $C_{\gamma 0}$, respectively. The concentration-distance curve was schematically drawn as shown in Fig. 1. The distances from the Matano interface to the $\beta / \alpha$ interface and the $\beta / \gamma$ interface are $r_{\alpha}$ and $r_{\gamma}$, respectively. When the relation $r_{\alpha}=2 \gamma_{\alpha} \sqrt{\overline{D_{\beta} t}}$ and $r_{\gamma}=2 \gamma_{\gamma} \sqrt{D_{\beta} t}$ are held, $W_{\beta}=\left|r_{\gamma}-r_{\alpha}\right|=2 \sqrt{D_{\beta} t}\left|\gamma_{\gamma}-\gamma_{\alpha}\right|$ results:

Then

$$
W_{\beta}^{2} / t=k_{\beta}^{2}=4\left(\gamma_{\gamma}-\gamma_{\alpha}\right)^{2} D_{\beta}
$$

where $W_{\beta}, D_{\beta}$ and $k_{\beta}$ are the width, the interdiffusion 1974 Vol. 15 
coefficient and the rate constant of growth of the $\beta$ phase layer, respectively.

Now, suppose that the relation $4\left(\gamma_{\gamma}-\gamma_{\alpha}\right)^{2}=$ $g_{0} \exp \left(-Q_{g} / R T\right)$ is satisfied. Then, from eq. (1),

$$
\begin{gathered}
k_{0 \beta}^{2} \exp \left(-Q_{k_{\beta}}^{2} / R T\right)=g_{0} \exp \left(-Q_{g} / R T\right) D_{0 \beta} \\
\times \exp \left(-Q_{D_{\beta}} / R T\right) .
\end{gathered}
$$

Then, eq. (2) is held:

$$
Q_{k_{\beta}^{2}}^{2}=Q_{D_{\beta}}+Q_{g} .
$$

Equation (2) shows that the activation energy for the layer growth of the $\beta$ phase is equal to a sum of $Q_{D_{s}}$ (the activation energy for an interdiffusion) and $Q_{g}$ (the energy introduced from the temperature dependence of $\left.4\left(\gamma_{\gamma}-\gamma_{\alpha}\right)^{2}\right)$. In short, the activation energy difference mentioned in the first section of this paper is attributable to the temperature dependence of $4\left(\gamma_{\gamma}-\right.$ $\left.\gamma_{\alpha}\right)^{2}$.

$\gamma_{\gamma}$ and $\gamma_{\alpha}$ are obtained as follows ${ }^{(5)(6)}$ :

Fick's second law is applied to the phases of $\alpha, \beta$ and $\gamma$, and eqs. (3) are introduced.

$$
\left.\begin{array}{ll}
\partial C / \partial t=D_{\alpha} \partial^{2} C / \partial x^{2} & \text { for } x<r_{\alpha} \\
\partial C / \partial t=D_{\beta} \partial^{2} C / \partial x^{2} & \text { for } r_{\alpha}<x<r_{\gamma} \\
\partial C / \partial t=D_{\gamma} \partial^{2} C / \partial x^{2} & \text { for } x>r_{\gamma}
\end{array}\right\} .
$$

For convenience' sake, the interdiffusion coefficients for the respective phases are assumed to be independent of composition. Considering the initial conditions and the boundary conditions shown by eqs. (4) and (5), respectively:

$$
\begin{gathered}
C=C_{\alpha 0} \quad \text { at } t=0 \text { and } x<0 \\
C=C_{\gamma 0} \quad \text { at } t=0 \text { and } x>0 \\
C=C_{\alpha \beta} \quad \text { at } x=r_{\alpha-\alpha} \\
C=C_{\beta \alpha} \text { at } x=r_{\alpha-\beta} \\
C=C_{\beta \gamma} \text { at } x=r_{\gamma-\beta} \\
C=C_{\gamma \beta} \quad \text { at } x=r_{\gamma-\gamma}
\end{gathered}
$$

The solutions of eqs. (3) are

$$
\begin{aligned}
& C=C_{\alpha 0}-B_{\alpha}\left[1+\operatorname{erf}\left(\frac{x}{2 \sqrt{D_{\alpha}} t}\right)\right] \quad \text { for } x<r_{\alpha}, \\
& C=A-B_{\beta} \operatorname{erf}\left(\frac{x}{2 \sqrt{D_{\beta} t}}\right) \quad \text { for } r_{\alpha}<x<r_{\gamma}, \\
& C=C_{\gamma 0}+B_{\gamma}\left[1-\operatorname{erf}\left(\frac{x}{2 \sqrt{D_{\gamma} t}}\right)\right] \quad \text { for } x>r_{\gamma} .
\end{aligned}
$$

Considering the flows of atoms at the $\beta / \alpha$ and $\beta / \gamma$ boundaries, respectively, eqs. (7) will be held approximately:

$$
\begin{aligned}
\left(C_{\alpha \beta}-\right. & \left.C_{\beta \alpha}\right) \frac{d r_{\alpha}}{d t}=J_{\alpha \rightarrow \beta}-J_{\beta \rightarrow \alpha} \\
& =-D_{\alpha}\left(\frac{\partial C}{\partial x}\right)_{r_{x-\alpha}}+D_{\beta}\left(\frac{\partial C}{\partial x}\right)_{r_{\alpha-\beta}}, \\
\left(C_{\beta \gamma}-\right. & \left.C_{\gamma \beta}\right) \frac{d r_{\gamma}}{d t}=J_{\beta \rightarrow \gamma}-J_{\gamma \rightarrow \beta} \\
& =-D_{\beta}\left(\frac{\partial C}{\partial x}\right)_{r_{\gamma-\beta}}+D_{\gamma}\left(\frac{\partial C}{\partial x}\right)_{r_{\gamma-\gamma}} .
\end{aligned}
$$

The above equations lead to

$$
\left.\begin{array}{c}
\frac{e^{-\gamma_{\alpha}{ }^{2} \varphi_{\alpha}}}{\gamma_{\alpha} \sqrt{\pi \varphi_{\alpha}}\left[1+\operatorname{erf}\left(\gamma_{\alpha} \sqrt{\left.\varphi_{\alpha}\right)}\right]\right.} \cdot \frac{\left(C_{\alpha 0}-C_{\alpha \beta}\right)}{\left(C_{\alpha \beta}-C_{\beta \alpha}\right)} \\
-\frac{e^{-\gamma_{\alpha}{ }^{2}}}{\gamma_{\alpha \alpha} \sqrt{\pi}\left[\operatorname{erf}\left(\gamma_{\gamma}\right)-\operatorname{erf}\left(\gamma_{\alpha}\right)\right]} \cdot \frac{\left(C_{\beta \alpha}-C_{\beta \gamma}\right)}{\left(C_{\alpha \beta}-C_{\beta \alpha}\right)} \\
=1=f\left(\gamma_{\gamma}, \gamma_{\alpha}\right) \\
\frac{e^{-\gamma_{\gamma}{ }^{2}}}{\gamma_{\gamma} \sqrt{\pi[}\left[\operatorname{erf}\left(\gamma_{\gamma}\right)-\operatorname{erf}\left(\gamma_{\alpha}\right)\right]} \cdot \frac{\left(C_{\beta \alpha}-C_{\beta \gamma}\right)}{\left(C_{\beta \gamma}-C_{\gamma \beta}\right)} \\
-\frac{e^{-\gamma_{\gamma} \varphi_{\gamma}}}{\gamma_{\gamma} \sqrt{\pi \varphi_{\gamma}}\left[1-\operatorname{erf}\left(\gamma_{\gamma} \sqrt{\left.\varphi_{\gamma}\right)}\right]\right.} \cdot \frac{\left(C_{\gamma \beta}-C_{\gamma 0}\right)}{\left(C_{\beta \gamma}-C_{\gamma \beta}\right)} \\
=1=g\left(\gamma_{\gamma}, \gamma_{\alpha}\right)
\end{array}\right\}
$$

where $\varphi_{\alpha}=D_{\beta} / D_{\alpha}$ and $\varphi_{\gamma}=D_{\beta} / D_{\gamma}$.

The simultaneous eq. (8) show that $\gamma_{\gamma}$ and $\gamma_{\alpha}$ are expressed as functions of the boundary concentrations of each phase and the ratios of the interdiffusion constant in the $\beta$ phase to those in the adjoining two phases $\left(\varphi_{\alpha}\right.$ and $\left.\varphi_{\gamma}\right)$. By solving the equations, the values of $\gamma_{\gamma}$ and $\gamma_{\alpha}$ are obtained. As seen in the equations, the error functions and exponential functions are included. Then the solution cannot be obtained analytically. However, it is possible to solve the equations graphically.

Now, the independent values of $\gamma_{\gamma}$ and $\gamma_{\alpha}$ which satisfy $f\left(\gamma_{\gamma}, \gamma_{\alpha}\right)=1$ and $g\left(\gamma_{\gamma}, \gamma_{\alpha}\right)=1$ are calculated numerically with a computer, and the values are traced on the $\gamma_{\gamma}-\gamma_{\alpha}$ co-ordinates as shown in Fig. 2. Thus, the values of $\gamma_{\gamma}$ and $\gamma_{\alpha}$ required are determined as the co-ordinate at the cross point of the two curves.

As mentioned above, the two activation energies for the interdiffusion and layer growth can be connected by using the value of $Q_{g}$ derived from the temperature dependence of $4\left(\gamma_{\gamma}-\gamma_{\alpha}\right)^{2}$. The following experimental study of the $\mathrm{Al}-\mathrm{Cu}$ system was used to ascertain the above relation.

\section{Experimental Procedure}

The $\gamma_{2}$ phase in the $\mathrm{Al}-\mathrm{Cu}$ system was prepared. The composition of the phase was 69.1 at $\% \mathrm{Cu}$. The alloy of this phase was plated with copper and supplied for a diffusion couple. The couple was sealed in an evacuated silica capsule and annealed in the temperature range of $640 \sim 759^{\circ} \mathrm{C}$. After the anneal, the cap-

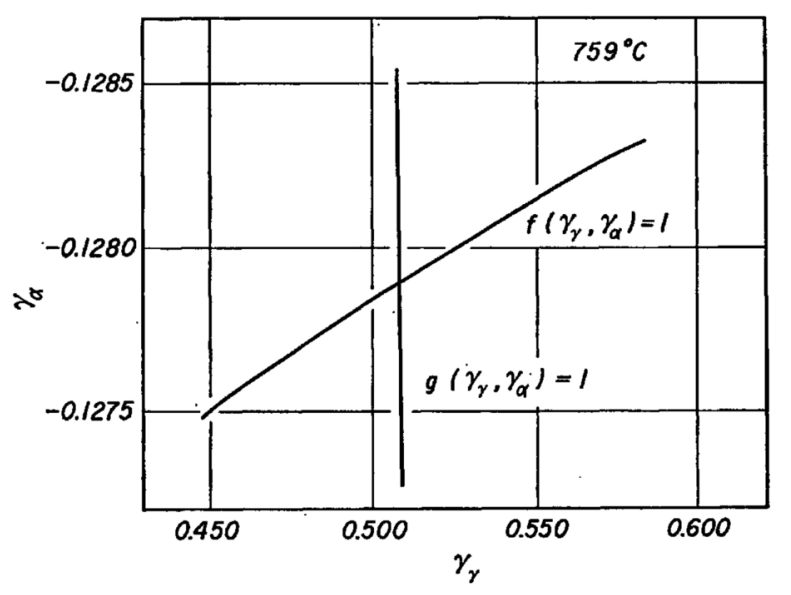

Fig. 2 Graph of the functions $f\left(\gamma_{\gamma}, \gamma_{\alpha}\right)=1$ and $g\left(\gamma_{\gamma}, \gamma_{\alpha}\right)=1$ numerically obtained at $759^{\circ} \mathrm{C}$. 


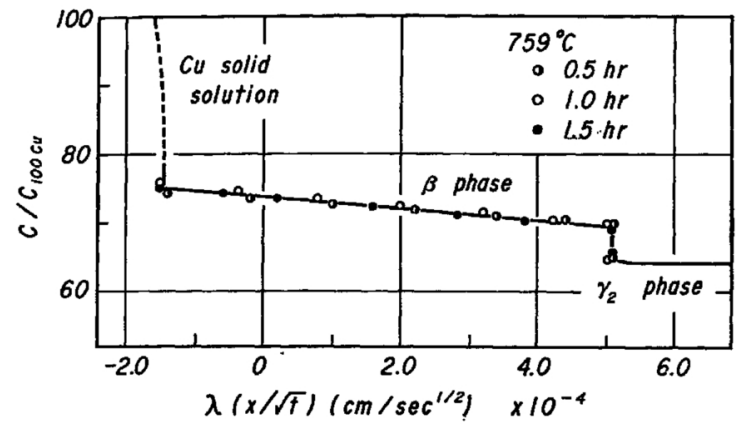

Fig. 3 Typical concentration-penetration curve for the diffusion zone at $759^{\circ} \mathrm{C}$.

sule was quenched in water. Then the couple was sectioned, polished and analyzed by use of an electron probe micro-analyzer with $\mathrm{Cu}-K_{\alpha}$ radiation. From the intensity of the radiation, the penetration curve was obtained as shown in Fig. 3. The relative intensity of the radiation was corrected with standard samples.

\section{Experimental Results and Discussion}

\section{Growth of the $\beta$ phase}

A typical example of a penetration curve for the couple annealed is shown in Fig. 3. The vertical axis is normalized as the molar concentration of copper. The horizontal axis represents the $x / \sqrt{t}$. As shown in the figure, the greater part of the diffusion zone was occupied by the $\beta$ phase and a fairly small amount of copper is dissolved in the $\gamma_{2}$ phase. A very small amount of copper solid solution was also developed, but the solubility limit of aluminum in the solution was not determined because of the very narrow width of the solid solution, and an unavoidable error must be introduced for the analysis of the concentration. The concentration at the solubility limit was taken from the Al-Cu phase diagram. This is shown with a broken line in Fig. 3.

In Fig. 4 the time dependence of the $\beta$ phase growth at each temperature is shown. It is shown clearly in the figure that the growth obeys a parabolic rate law. In Fig. 5 the temperature dependence of the growth rate of the phase is shown. Again an excellent linear relation exists clearly in the Arrhenius plot. From the gradient of the line, the activation energy for the layer

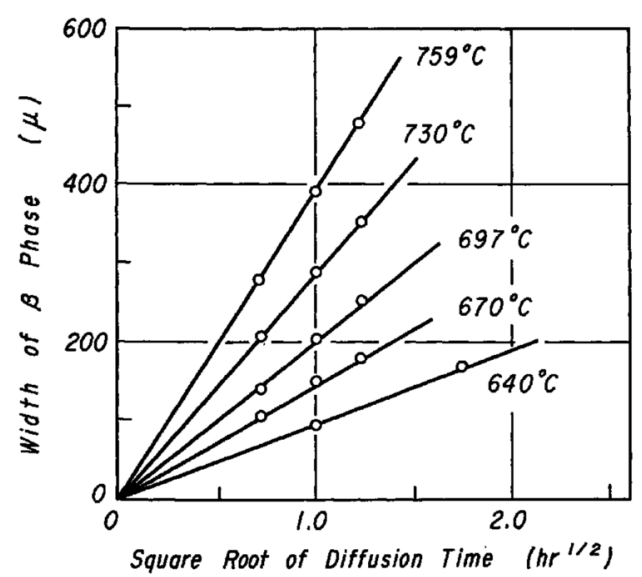

Fig. 4 Growth curves of the $\beta$ phase layer.

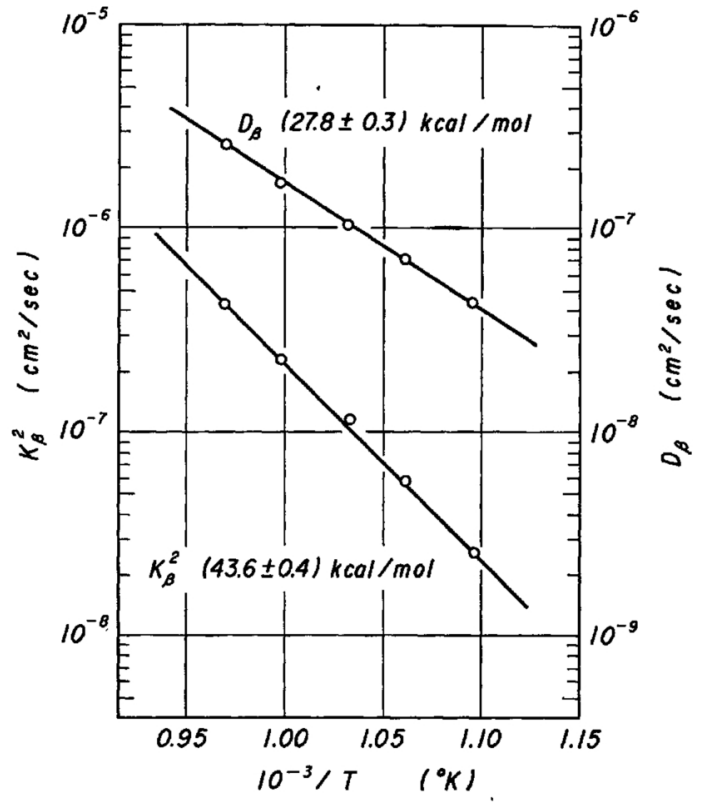

Fig. 5 Temperature dependence of $k_{\beta}^{2}$ and $D_{\beta}$.

growth was obtained by the least squares method. The value was $43.6 \pm 0.4 \mathrm{kcal} / \mathrm{mol}$. The error limit refers to the probable deviation.

\section{Interdiffusion coefficient}

The interdiffusion coefficient of the $\beta$ phase $\left(D_{\beta}\right)$ was determined according to Heumann's method ${ }^{(7)}$ assuming that the concentration profile within the $\beta$ phase is approximately linear. In Fig. 5 the temperature dependence of the interdiffusion coefficient is also shown and a fairly good linear relation is found as well. The activation energy for interdiffusion was $27.8 \pm 0.3 \mathrm{kcal} / \mathrm{mol}$. Asundi et al. ${ }^{(8)}$ obtained the activation energy for interdiffusion in the $\beta$ phase. They measured the movement of the phase boundary of the phase and calculated the energy with Wagner's equation to be $27.5 \mathrm{kcal} / \mathrm{mol}$. These two results coincide with each other.

The difference between the activation energies for the layer growth and interdiffusion of this phase is about $16 \mathrm{kcal} / \mathrm{mol}$.

\section{Calculation of $\mathbf{4}\left(\gamma_{\gamma}-\gamma_{\alpha}\right)^{2}$}

The concentration of each phase boundary plays an important role in the calculation of the value of $4\left(\gamma_{\gamma}-\gamma_{\alpha}\right)^{2}$. The concentration obtained from the present study is shown in Fig. 6 with open circles and the equilibrium concentration in the phase diagram ${ }^{(9)}$ is shown by dotted lines in the figure. The concentration at the solubility limit of the $\gamma_{2}$ phase obtained in this study coincides nearly with the equilibrium value, whereas the composition range of the $\beta$ phase shifts to the copper side by about one atomic per cent compared with the diagram.

The values used for the present calculation are tabulated in Table 1 . The interdiffusion coefficients in the $\alpha$ and $\gamma_{2}$ phases, respectively, were cited from published papers ${ }^{(1)(10)}$ and $\varphi_{\alpha}$ and $\varphi_{\gamma}$ were calculated with those data in the papers.

Figure 2 shows an instance of the numerically ob- 


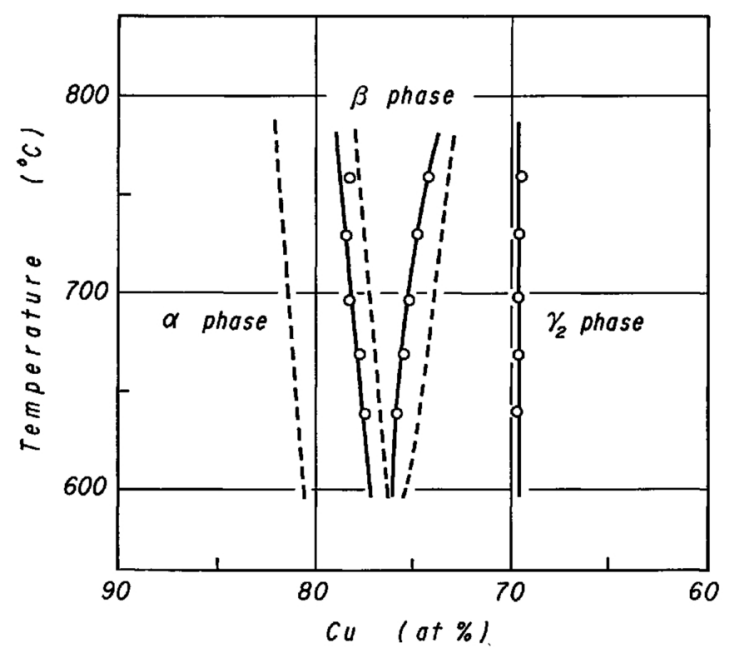

Fig. 6 Boundary compositions at the phase interface. Dotted lines represent the equilibrium composition in the phase diagram.

Table 1 The values used for the present calculation.

\begin{tabular}{|c|c|c|c|c|c|c|c|}
\hline & $\begin{array}{l}C_{\alpha \beta}-C_{\beta \circ} \\
(\mathrm{mol} \%)\end{array}$ & $\begin{array}{l}C_{\alpha 0}-C_{\alpha \beta} \\
(\mathrm{mol} \%)\end{array}$ & $\begin{array}{l}C_{\beta \alpha}-C_{\beta \gamma} \\
(\mathrm{mol} \%)\end{array}$ & 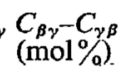 & $\begin{array}{c}C_{\gamma \beta}-C_{\gamma 0} \\
(\mathrm{~mol} \mathrm{\%} \%)\end{array}$ & $\varphi_{\alpha}$ & $\varphi_{\gamma}$ \\
\hline $759^{\circ} \mathrm{C}$ & 3.9 & 21.5 & 4.6 & 5.1 & 0.5 & 930 & 2.0 \\
\hline $730^{\circ} \mathrm{C}$ & 3.6 & 21.7 & 4.0 & 5.8 & 0.5 & 1200 & 2.1 \\
\hline $697^{\circ} \mathrm{C}$ & 3.4 & 22.1 & 3.3 & 6.1 & 0.7 & 1600 & 2.4 \\
\hline $670^{\circ} \mathrm{C}$ & 3.7 & 22.3 & 2.6 & 6.2 & 0.8 & 2000 & 2.5 \\
\hline $640^{\circ} \mathrm{C}$ & 3.9 & 22.5 & 1.7 & 6.7 & 0.8 & 2900 & 2.8 \\
\hline
\end{tabular}

tained traces of the functions $f\left(\gamma_{\gamma}, \gamma_{\alpha}\right)=1$ and $g\left(\gamma_{\gamma}, \gamma_{\alpha}\right)=1$ at $759^{\circ} \mathrm{C}$. Figure 7 shows the temperature dependence of $4\left(\gamma_{\gamma}-\gamma_{\alpha}\right)^{2}$, and its Arrhenius plot shows a straight line. The activation energy $\left(Q_{g}\right)$ was obtained from the gradient of the line to be $18.3 \pm 0.9$ $\mathrm{kcal} / \mathrm{mol}$. The sum of $Q_{D_{b}}$ and $Q_{g}$ was $46.1 \mathrm{kcal} / \mathrm{mol}$. On the other hand, the activation energy for the layer growth $\left(Q_{k_{\beta}^{2}}^{2}\right)$ was $43.6 \mathrm{kcal} / \mathrm{mol}$. Thus it is considered that the relation expressed by eq. (2) holds fairly well within an experimental error.

It is important here to discuss the details of the temperature dependence of $4\left(\gamma_{\gamma}-\gamma_{\alpha}\right)^{2}$. As mentioned already, it is a function of $\varphi_{\alpha}, \varphi_{y}$ and the concentration of each phase boundary. If the simultaneous eq. (8) could solved analytically, it would be clear how the above variables contribute to the value of $4\left(\gamma_{\gamma}-\right.$

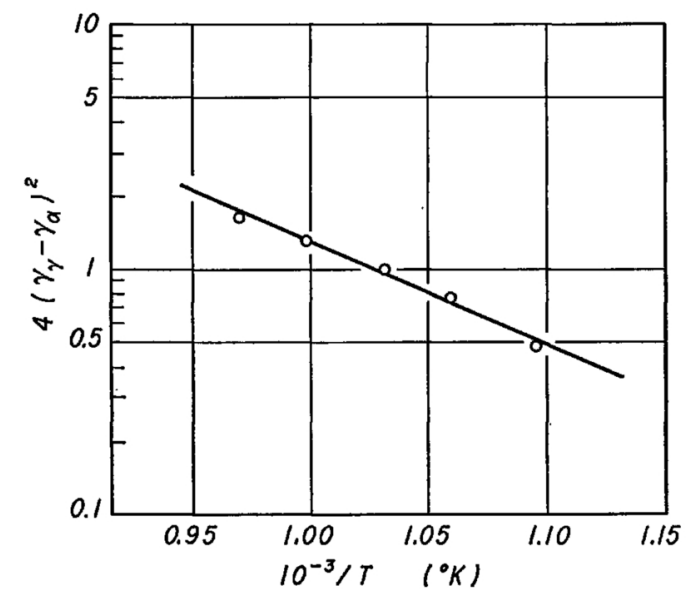

Fig. 7 Temperature dependence of $4\left(\gamma_{\gamma}-\gamma_{\alpha}\right)^{2}$.

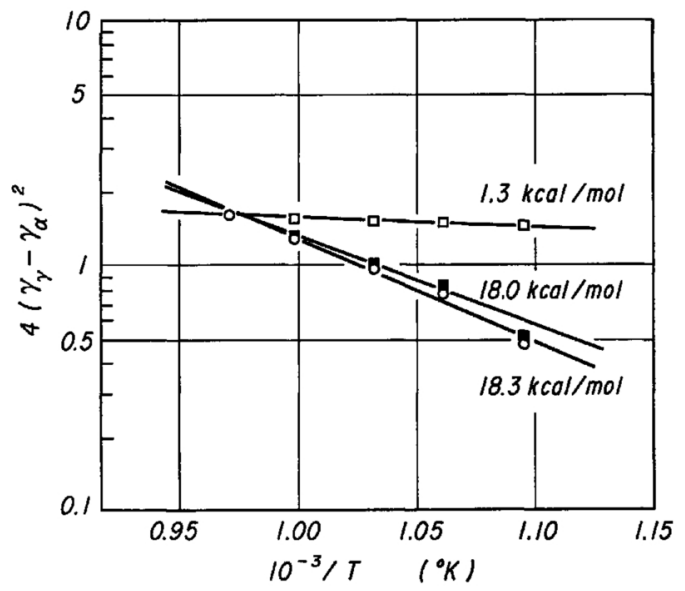

Fig. 8 The details of the temperature dependence of $4\left(\gamma_{\gamma}-\gamma_{\alpha}\right)^{2}$.

$\square$ : the case in which merely $\varphi_{\alpha}$ and $\varphi_{\gamma}$ are variable.

: the case in which merely the concentration is variable.

$\bigcirc$ : the case in which all variables are changed.

$\left.\gamma_{\alpha}\right)^{2}$. However, the solution can not be analytically obtained. Accordingly, the graphical analysis was also attempted. Two conditions are used in this case; that is, (1) $\varphi_{\alpha}$ and $\varphi_{\gamma}$ are variable and the concentration at each phase boundary is constant, and (2) $\varphi_{\alpha}$ and $\varphi_{\gamma}$ are constant and the concentration is variable. Then the value of $4\left(\gamma_{\gamma}-\gamma_{\alpha}\right)^{2}$ can be calculated following the procedures (1) and (2). The result is shown in Fig. 8. The symbol $\square$ corresponds to the case (1) where merely $\varphi_{\alpha}$ and $\varphi_{\gamma}$ are variable. From the figure it may be seen clearly that the value of $4\left(\gamma_{\gamma}-\gamma_{\alpha}\right)^{2}$ has a very small temperature dependency.

The symbol $\square$ indicates the result from case (2) where the concentration is variable. The result nearly coincides with the case in which all variables were changed (symbol $\bigcirc$ ). From these results it should be emphasized that the principal factor controlling the temperature dependence of $4\left(\gamma_{\gamma}-\gamma_{\alpha}\right)^{2}$ is the temperature dependence of the concentration at each phase boundary. Especially, the temperature dependence of the concentration range of the $\beta$ phase $\left(C_{\beta \alpha}-C_{\beta \gamma}\right)$ must be the most important factor as can be easily seen from Fig. 6 .

The present results also explain the results of the interdiffusion study on the systems $\mathrm{Al}-\mathrm{Cu}^{(1)}$ and $\mathrm{Al}-\mathrm{Mg}^{(2)}$. With these systems, several intermediate phases are formed. Among the phases one which has no temperature dependence of the composition range shows that $Q_{k_{\beta}}$ and $Q_{D_{\beta}}$ are almost identical. On the contrary, the phases which have a relatively large temperature dependence of the composition range show the difference between the two activation energies.

\section{Summary}

This investigation was carried out for the case where the layer growth of the intermediate phase formed in a poly-phase diffusion couple was controlled by the process of volume diffusion and the activation energy 
for the layer growth differed from that of the interdiffusion in that phase.

The results can be summarized as follows:

(1) It was quantitatively shown that the difference between these two activation energies originated from the temperature dependence of the homogeneity range of the phase and from the concentration range of adjoining two-phase areas, especially from the former when the range depends strongly on temperature.

(2) In the interdiffusion on the $\beta$ phase of an $\mathrm{Al}-\mathrm{Cu}$ system the activation energies were $27.8 \mathrm{kcal} /$ mol for the interdiffusion $\left(Q_{D_{\beta}}\right), 43.6 \mathrm{kcal} / \mathrm{mol}$ for the layer growth $\left(Q_{k_{\beta}^{2}}\right)$, and the energy derived from the temperature dependence of each phase boundary concentration $\left(Q_{g}\right)$ was $18.3 \mathrm{kcal} / \mathrm{mol}$. The sum of $Q_{D_{k}}$ and $Q_{g}$ was $46.1 \mathrm{kcal} / \mathrm{mol}$. The value thus added coincided with that of $Q_{k_{1 j}^{2}}$.

(3) The activation energy for the layer growth is identified with that for the interdiffusion in the case where the phase has no temperature dependence of the composition range. On the contrary, the two kinds of activation energies must be dissimilar each other when the composition range of the phase varies with temperature.

\section{REFERENCES}

(1) Y. Funamizu and K. Watanabe: Trans. JIM, 12 (1971), 147.

(2) Y. Funamizu and K. Watanabe: Trans. JIM, 13 (1972), 278.

(3) K. Hirano and Y. Ipposhi: J. Japan Inst. Metals, 32 (1968), 815.

(4) G. V. Kidson: J. Nucl. Mater., 3 (1961), 21.

(5) G. B. Gibbs: J. Nucl. Mater., 20 (1966), 303.

(6) W. Jost: Diffusion in Solids, Liquids and Gases, Academic Press Inc., New York, (1960), p. 74.

(7) Th. Heumann: Z. Phys. Chem., 201 (1952), 168.

(8) M. K. Asundi and D. R. West: Trans. Met. Soc. AIME, 230 (1964), 428.

(9) M. Hansen and K. Anderko: Constitution of Binary Alloys, McGrow Hill, New York, (1958), p. 85.

(10) H. Oikawa, T. Obara and S. Karashima: Met. Trans., 1 (1970), 2969. 